\title{
Searches for Heavy Di-boson Resonances with the ATLAS Detector
}

\section{Ilic Nikolina ${ }^{1,2}$ on behalf of the ATLAS Collaboration}

1. University of Toronto \& Institute of Particle Physics

27 King's College Circle, Toronto, Ontario, M5S 1A1, Canada

2. Radboud University

Houtlaan 4, 6525 XZ Nijmegen, Netherlands

E-mail: NikolinaIlic@cern.ch

The ATLAS collaboration has performed searches for new heavy resonances decaying to pairs of vector bosons and Higgs bosons with $36 \mathrm{fb}^{-1}$ and $139 \mathrm{fb}^{-1}$ of data delivered by the Large Hadron Collider. These proceedings present selected analyses in which new particles such as new scalar particles, $W^{\prime}$ and $Z^{\prime}$ bosons and gravitons decay to various combinations of vector and Higgs bosons. 


\section{Introduction}

Many beyond the Standard Model (SM) theories predict the existence of new, TeV-scale resonances that can decay to a pair of vector bosons ( $W$ and $Z$ ) or Higgs bosons. These theories include two-Higgs-Doublet models (2HDM), Randall-Sundrum (RS) models, Grand Unified theories or simplified models such as the Heavy Vector Triplet (HVT) framework. The Large Hadron Collider delivered $36 \mathrm{fb}^{-1}$ of data in 2015 and 2016, and $139 \mathrm{fb}^{-1}$ up to 2018. The ATLAS Collaboration has used these data sets to search for vector boson resonances decaying to many combinations of leptons particles. These proceedings report on a subset of the results that include heavy resonance decays to two vector bosons, a vector boson a Higgs boson and two Higgs Bosons, that subsequently decay to various final states.

The ATLAS detector [1] consists of several subdetectors which are well equipped to measure these final states. The inner detector, consisting of the Pixel Detector, Semiconductor Tracker and Transition Radiation Tracker, measures the momentum and charge of particles by analyzing the tracks they produce. The electromagnetic calorimeter, called the Liquid Argon calorimeter, measures energy deposits left by electromagnetically interacting particles. The hadronic calorimeter, known as the Tile Calorimeter, measures energy deposits created by hadrons. The outermost part of ATLAS consists of muon chambers that include Thin Gap Chambers, Resistive Plate Chambers, Monitored Drift Tubes, and Cathode Strip chambers, for muon detection. Neutrinos do not interact with the detector, but their presence can be deduced by inferring the amount of energy missing in the transverse direction.

\section{Two Vector Bosons}

ATLAS performed a search for Beyond Standard Model (BSM) particle decaying two vector bosons ( $W$ or $Z$ ), which subsequently decay to quarks that are reconstructed as jets in the ATLAS detector using $139 \mathrm{fb}^{-1}$ of data [2]. The possible new particles considered were spin-1 $W$ ' and $Z$ ' models as predicted by the HVT framework, and spin-2 gravitons as predicted by RS models. Within the HVT framework, two benchmark models are used, referred to as Model A and Model B. In Model A, the branching fractions of the $W^{\prime} / Z^{\prime}$ to fermions and SM gauge bosons are comparable, as in models with an extended gauge symmetry. In Model B, the couplings of the $W^{\prime} / Z^{\prime}$ particles to fermions are supressed, as in models like the minimal composite Higgs model.

The advantage of considering the hadronic quark decay modes is the high branching fraction (67\% for $W$ boson decays and $70 \%$ for $Z$ boson), while the disadvantage is the large backgrounds from SM multi-jet events. Since these multi-jet events are poorly reconstructed in Monte Carlo simulations, they are modelled by a parametric functional fit to data. When the vector bosons have a high momentum, their decay products are very collimated. Thus, instead of using two small-radius $(R=0.4)$ jets initiated by each quark from the $W$ or $Z$ decay, the analysis uses large radius $(R=1)$, that encompass both quarks from $W$ or $Z$ decay. Since the calorimeter clusters used in the construction of large radius jets are close to the granularity limits of the calorimeter, angular track information is added to the cluster to improve the spatial resolution. Therefore, the clusters used in the large-radius jet construction, called Track-CaloCluters (TCC), utilize a combination of momentum and energy information from the calorimeter, and spatial information from the inner detector. A $W / Z$ boson tagger identifies whether large-radius jets were initiated by $W$ or $Z$ decays by utilizing substructure information 
within the jets. One of the most useful sub-structure variables, referred to as $D_{2}$ [3], utilizes 2- and 3-point energy correlation functions in order to identify which energy deposits in the large-radius jet are due to collinear deposits, and which are the result of soft radiation. Figure 1 shows the invariant mass distribution of two large radius jets originating from the two vector bosons. In the statistical fit, this kinematic variable is used to discriminate between the signal events and the SM background. The masses that are excluded by the statistical fit for various signal models are shown in Table 1. No excesses above the SM expectations are found.

\begin{tabular}{|lll|}
\hline Model & $\begin{array}{l}\text { Signal } \\
\text { Region }\end{array}$ & $\begin{array}{l}\text { Excluded Mass } \\
\text { (TeV) }\end{array}$ \\
\hline HVT model A & $W W / W Z$ & $1.3-2.9 / 1.3-3.4$ \\
\hline HVT model B & $W W / W Z$ & $1.3-3.1 / 1.3-3.6$ \\
Graviton & $W W$ & $1.1-1.6$ \\
\hline
\end{tabular}

Table 1: The mass ranges excluded, at a $95 \%$ confidence level, in a search for HVT $W^{\prime} / Z$ ' bosons, and the $R S$ Graviton [2].

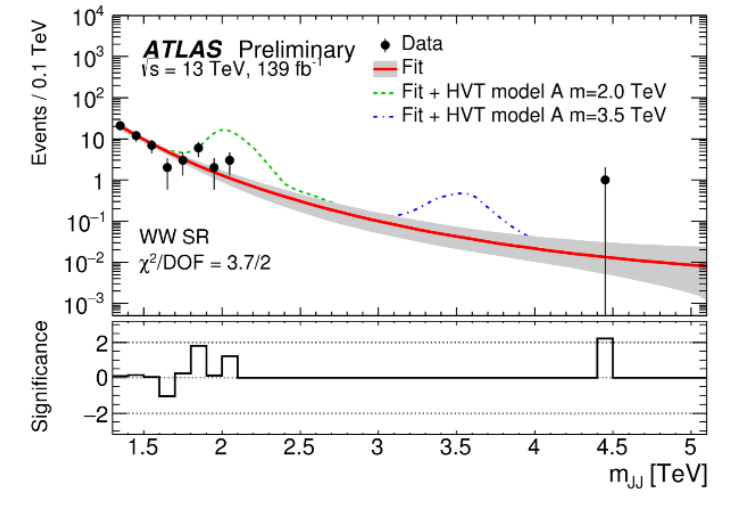

Figure 1: The invariant mass distribtuion of the large-radius di-jet system. The data-driven background estimation is shown in red, while the green and blue lines represent the HVT model A signal for masses of 2 and $3 \mathrm{TeV}$, respectively [2].

\section{A Vector Boson and Higgs Boson}

The ATLAS Collaboration has also searched for resonances decaying to a vector boson ( $W$ or $Z$ ) together with a SM Higgs boson using the $36 \mathrm{fb}^{-1}$ dataset [4]. The new particle searched for were $W^{\prime}$ and $Z$ ' as predicted by the HVT framework, as well as the CP-odd scalar boson $(A)$, as predicted by 2 HDM models. The search was performed in the case where the Higgs boson decayed to two $b$-quarks, and the vector boson decayed to leptons and neutrinos. Higgs boson decays to small- and large-radius jets were considered, and the jets were tagged as having been initiated by $b$-quarks. This tagging was performed by standard ATLAS $b$-tagging algorithms for the small radius jets, and by matching large radius jets to jets consisting of tracks that are tagged as originating from $b$-quarks. The mass or transverse mass of the Higgs and vector boson system is used to discriminate the signal from the leading backgrounds consisting of SM top quarks and $W$ bosons produced with jets. Dedicated control regions, depleted of signal are used to obtain normalization factors that correct the background MC simulation to the data in cases when small discrepancies between the two are present. Figure 2 (left) shows an example of the transverse mass in one of the regions used to search for an HVT signal. Figure 2 (right) shows an example of exclusion limit plots in the search for the scalar boson, $A$. Limits are set on $\mathrm{m}_{W^{\prime}}<2.67 \mathrm{TeV}(2.82 \mathrm{TeV})$ and $\mathrm{m}_{Z^{\prime}}<2.65 \mathrm{TeV}(2.83 \mathrm{TeV})$ in HVT Model A (Model B). For the 2HDM, masses below 1.3 TeV - 1.6 TeV are excluded, depending on the final signal region analysed. A $3.6(2.4) \sigma$ local (global) excess in found at a mass of $\mathrm{m}_{A}=440 \mathrm{GeV}$ in the associated $b$-production channel. 

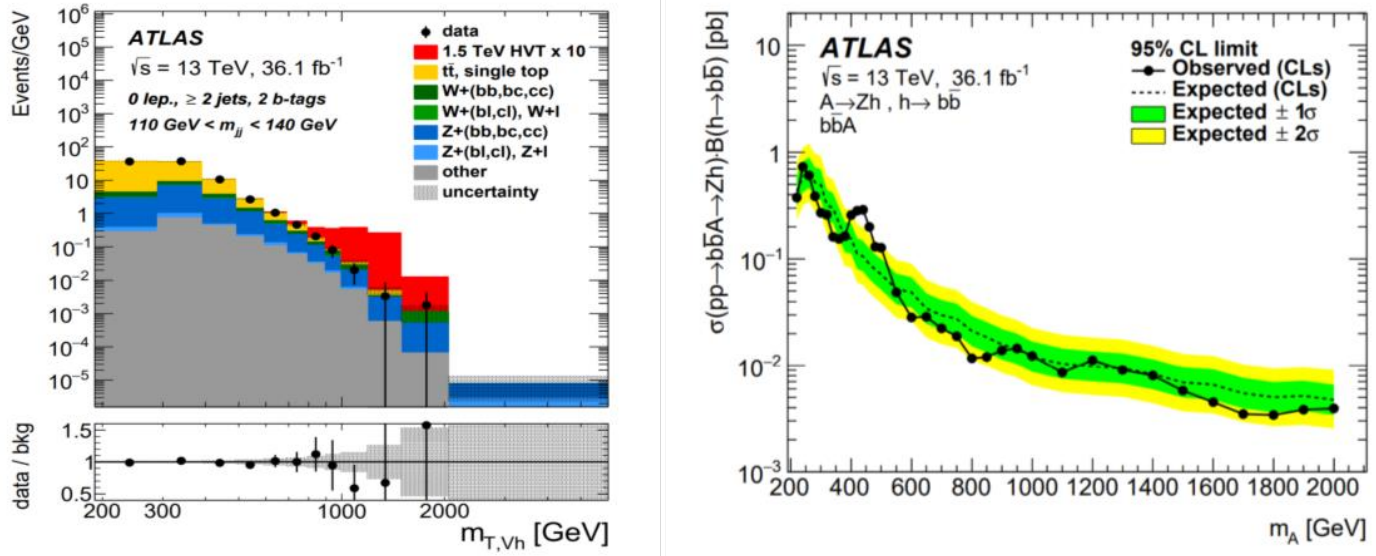

Figure 2. The transverse mass of the vector-boson and Higgs system for a $1.5 \mathrm{TeV} H \mathrm{HT}$ signal model and observed data and background prediction (left) [4]. The limit on the cross section times branching ratio at a 95\% confidence level in the search for a scalar particle, A (right) [4].

\section{Vector Boson Combinations}

Searches for di-boson resonances that are produced by the decay of a new particle in gluon-gluon fusion, vector boson fusion, or Drell-Yan production were performed with $36 \mathrm{fb}^{-1}$ data [5]. The various production modes explored are shown in Figure 3. The new particle signal models considered were the $W^{\prime}$ and $Z$ ' particles in HVT, the graviton as predicted by RS models, and a new heavy scalar singlet. Many decay modes of the bosons were considered, including fully hadronic, partially leptonic, fully leptonic and states with $b$-quarks. Signal regions to search for various decay modes were selected by making requirements on the number of leptons, jets and value of the missing transverse energy. The invariant mass or transverse invariant mass are used to discriminate the signal from standard model background processes. Figure 4 (left) shows an example of an exclusion plot for the HVT signal, in which the $W^{\prime}$ or $Z$ ' boson decays to two vector bosons or a vector boson and Higgs boson. In HVT model A (B), new heavy resonances are excluded below $<5.5 \mathrm{TeV}$ (4.5), while in RS models, gravitons are excluded if $m_{g}<2.3 \mathrm{TeV}$. These results were also combined with di-lepton searches to set limits on the leptonic, quark, and Higgs and vector bosons couplings to Higgs in the HVT models. Figure 4 (right) shows that in most regions of the phase space, these combinations provide improved limits with respect to the current constraints from precision electroweak measurements. The combination show that the constraints on HVT model A are stronger than ones derived for HVT model B due to the small fermion couplings in model B.
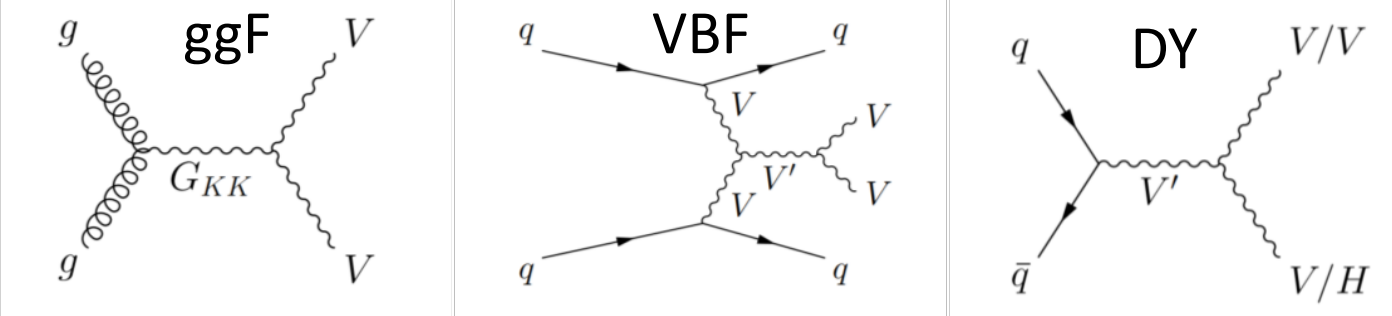

Figure 3: Production of vector bosons via gluon-gluon fussion ( $g g F)$ (left), vector boson fusion (VBF) (middle), and Drell-Yan production (DY) (right) [5]. 

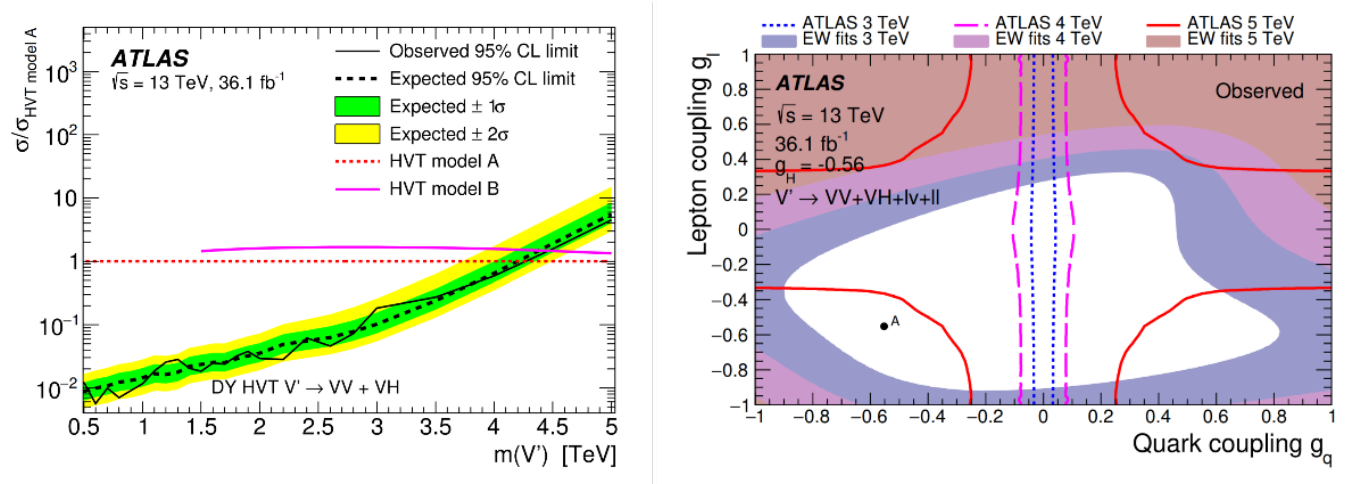

Figure 4: Limits on the cross section, at a 95\% Confidence Level, for a $W$ 'or $Z$ ', labelled $V$ ', decaying to two vector bosons or a vector boson and Higgs boson for the HVT model A (left) [5]. The limits on the lepton coupling versus quark coupling in the HVT framework for final states that include vector bosons and leptons (right). The lines represent the ATLAS limits at various mass points, with the couplings outside of the lines excluded for each $W^{\prime} / Z^{\prime}$ mass. The solid colors represent limits on the couplings from EW fits at various mass points [5].

\section{Two Higgs Bosons}

In the Standard Model, the production rate of two Higgs bosons is much lower than single Higgs production. However, di-Higgs production can be enhanced if the couplings to the Higgs boson are modified with respect to their SM values (non-resonant production), or if a new type of particle is produced that decays to two Higgs bosons (resonant production). The ATLAS collaboration has performed searches for non-resonant di-Higgs production and resonant di-Higgs production when produced through the decay of a $2 \mathrm{HDM}$ particle, RS graviton or a heavy scalar particle, $S$. The ATLAS analyses explored regions in which the Higgs bosons decayed to $4 b$-quarks [6], $2 b$-quarks and $2 W$ bosons [7], $4 \mathrm{~W}$ bosons [8], $2 b$-quarks and 2 tau leptons [6], and $2 b$-quarks and 2 photons [6]. Figure 5 (left) shows the limit on the cross-section times branching ratio for several of these final decay modes. A small deficit with respect to the background-only prediction is observed. Figure 5 (right) shows the Boosted Decision Tree score used as the discriminating variable to differentiate the Higgs bosons decay to $2 b$-quarks and 2 tau leptons from its SM background processes. The plot shows that the largest background is from SM top events, which are normalized from a control region enriched in top events. Another large background to the search for di-Higgs bosons decaying to $2 b$-quarks and 2 tau leptons consists of jets and leptons mis-identified as tau leptons. Since this background is difficult to predict in MC simulation, it is estimated from a data-driven method, called the Fake Factor method. This method relies on estimating the probability, or Fake Factor, of a jet or lepton faking a tau-lepton in control regions that are rich in multi-jet, $W+$ jet and top events. The probability is then applied to the regions that are rich is background events (control regions), in order to estimate the amount of multi-jet, $W+$ jet and top events that produce fake tau leptons in the signal regions. 

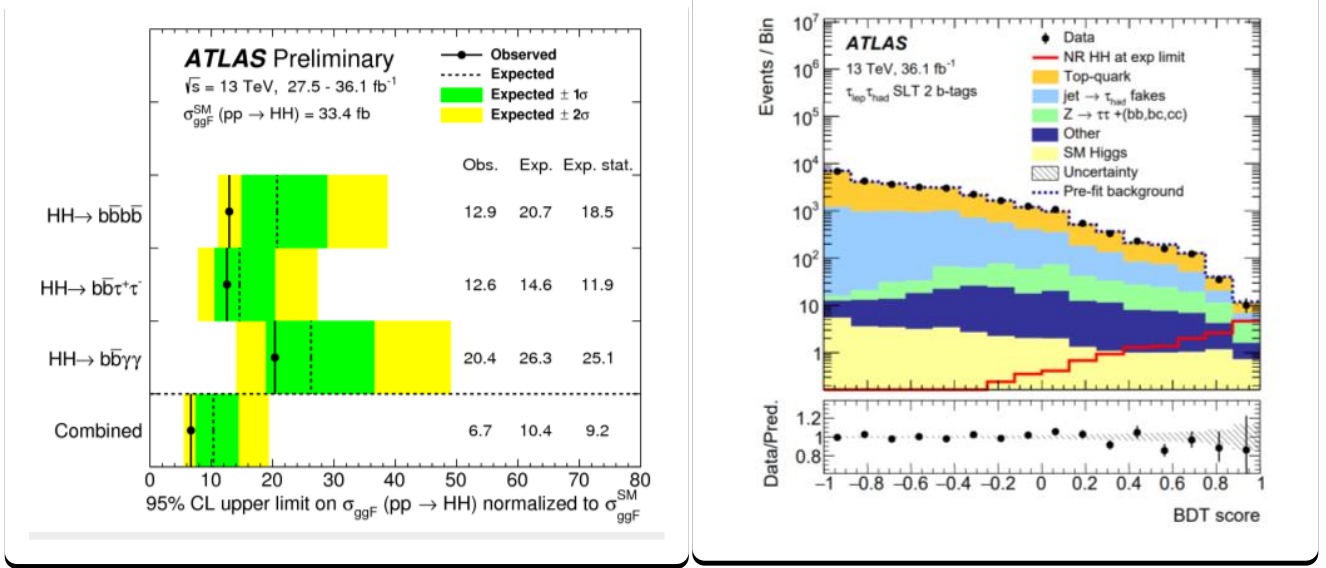

Figure 5: The upper limit on the gluon-gluon fusion cross section times branching ratio for di-Higgs production in various decay modes (left) [6]. The BDT score used as the discriminating variable in the channel in which two Higgs bosons decay to two b-quarks and two tau leptons (right) [6].

\section{Summary}

Searches for a heavy resonance decaying to a pair of vector bosons, a vector boson and Higgs boson, and two Higgs bosons were presented. Significant excesses were not found in any of the searches, and limits on cross sections and masses for scalar particles, spin-1 $W^{\prime}$ and $Z$ 'models as predicted in the HVT framework, and spin-2 gravitons as predicted by RS models are presented.

\section{References}

[1] ATLAS Collaboration, The ATLAS Experiment at the CERN Large Hadron Collider, 2008 JINST 3 S0800.

[2] ATLAS Collaboration, Search for diboson resonances in hadronic final states in $139 \mathrm{fb}^{-1}$ of pp collisions at $\sqrt{s}=13 \mathrm{TeV}$ with the ATLAS detector, ATLAS-CONF-2019-003, https://cds.cern.ch/record/2667227.

[3] ATLAS Collaboration, Identification of boosted, hadronically decaying $W$ bosons and comparisons with ATLAS data taken at $\sqrt{s}=8 \mathrm{TeV}$, Eur. Phys. J. C 76(3) (2016) 1-47.

[4] ATLAS Collaboration, Search for heavy resonances decaying into a $W$ or $Z$ boson and a Higgs boson in final states with leptons and b-jets in $36 \mathrm{fb}^{-1}$ of $\sqrt{s}=13 \mathrm{TeV}$ pp collisions with the ATLAS detector, JHEP 03 (2018) 174.

[5] ATLAS Collaboration, Combination of searches for heavy resonances decaying into bosonic and leptonic final states using $36 \mathrm{fb}^{-1}$ of proton-proton collision data at $\sqrt{\mathrm{s}}=13 \mathrm{TeV}$ with the ATLAS Detector, Phys. Rev. D 98 (2018) 052008.

[6] ATLAS Collaboration, Combination of searches for Higgs boson pairs in pp collisions at $13 \mathrm{TeV}$ with the ATLAS experiment, ATLAS-CONF-2018-043, https://cds.cern.ch/record/2638212.

[7] ATLAS Collaboration, Search for Higgs boson pair production in the $b b W W$ decay mode at $\sqrt{s}=13$ TeV with the ATLAS Detector, JHEP 04 (2019) 092.

[8] ATLAS Collaboration, Search for Higgs boson pair production in the $W W W W$ decay channel using ATLAS data recorded at $\sqrt{s} 13 \mathrm{TeV}$, JHEP 05 (2019) 124. 